\title{
Effect of periodontal treatment on peak serum levels of inflammatory markers
}

\author{
Adnan Ali Almaghlouth • Norbert Cionca • José Antonio Cancela • \\ Fabien Décaillet • Delphine S. Courvoisier • Catherine Giannopoulou • \\ Andrea Mombelli
}

Received: 21 May 2013 / Accepted: 13 January 2014 /Published online: 23 January 2014

(C) Springer-Verlag Berlin Heidelberg 2014

\begin{abstract}
Background and objective Some subjects with untreated periodontitis exhibit elevated levels of distinct inflammatory markers in serum. The aim of the study was to assess whether nonsurgical periodontal therapy changes the levels of these markers and lowers these peaks.

Methods Forty periodontally diseased subjects received nonsurgical periodontal therapy (full-mouth scaling and root planing within $48 \mathrm{~h}$ ) with either adjunctive systemic amoxicillin and metronidazole $(n=19)$ or placebo $(n=21)$. Serum samples, obtained at baseline (BL) and 3 months after treatment (M3), were evaluated for 15 cytokines and 9 acute-phase proteins using the Bio-Plex bead array multianalyte detection system. For each analyte, peak values were defined as greater than the mean +2 standard deviations (SD) of measurements found in 40 periodontally healthy persons. Proportions were compared using Fisher's exact test.

Results At M3, a significantly better primary clinical outcome (persisting pockets of $>4 \mathrm{~mm}$ with bleeding on probing) was obtained in patients treated with scaling and root planing plus antibiotics compared to those receiving placebo $(3.3 \pm 5.1 \mathrm{vs.}$ $6.8 \pm 7.8$ pockets per patient, $p<0.05$ ). The levels of cytokines and acute-phase proteins of periodontitis patients were usually below the mean +2 SD threshold of healthy persons.
\end{abstract}

One-sentence summary Nonsurgical periodontal treatment with or without antibiotics reduced peak levels of several inflammatory markers in serum of subjects with periodontitis.

A. A. Almaghlouth · N. Cionca · J. A. Cancela · F. Décaillet ·

C. Giannopoulou $\cdot$ A. Mombelli $(\bowtie)$

Division of Oral Physiopathology and Periodontics, School of Dental

Medicine, University of Geneva, Rue Barthélemy-Menn 19,

1205 Geneva, Switzerland

e-mail: andrea.mombelli@unige.ch

D. S. Courvoisier

Department of Psychology, Harvard University, Cambridge, MA, USA
However, values above threshold were found in some individuals. Eleven patients showed a peak value of one analyte, and seven patients showed two peaks. In the remaining 12 patients, between three and ten analytes showed peak values. Therapy greatly reduced the number of subjects with four or more peaks (BL, 11 subjects; M3, 1 subject, $p=0.003$ ). With regards to the reduction of peaks, no specific benefit of adjunctive antibiotics could be seen.

Conclusion Subjects with untreated periodontitis may show high peaks for several inflammatory markers in serum simultaneously. Nonsurgical periodontal treatment with or without antibiotics reduced most of these peak levels.

Keywords Nonsurgical periodontal therapy · Antibiotics · Serum $\cdot$ Inflammatory mediators

\section{Introduction}

Periodontitis is associated with elevated serum inflammatory markers (for review, see [1]). This suggests that periodontitis may have an impact on systemic health and insinuates that efforts to maintain or restitute periodontal health may contribute to systemic health. Studies have indicated that biological markers of systemic conditions like atherosclerosis are more prevalent in periodontitis patients than in age- and gendermatched controls [2]. However, the currently available data on periodontal systemic associations are rather heterogeneous [3], and the evidence that periodontal therapy has an impact on systemic health is limited [4]. A few studies have tried to elucidate whether clinically successful periodontal treatment can reduce the levels of serological markers and have yielded inhomogeneous results. A preliminary intervention study indicated that standard nonsurgical therapy (scaling and root planing (SRP)) might be able to change levels of IL-6 and C-reactive protein [5]. In a comparative study, SRP reduced 
levels of IL-6 and C-reactive protein and, if local antibiotics were added, also total and LDL cholesterol in serum [6]. In another study, however, periodontal therapy improved clinical and microbiological parameters but did not influence the levels of serum analytes [7]. In patients with chronic periodontitis and known coronary artery disease, SRP reduced high-sensitivity C-reactive proteins (hsCRPs) and white blood cell counts; however, tumor necrosis factor alpha (TNF- $\alpha$ ) levels showed no statistically significant reduction [8]. In patients with metabolic syndrome, CRP levels decreased after nonsurgical periodontal therapy, with or without adjunctive antibiotics [9]. A systematic review has concluded that plasma CRP in periodontitis is elevated, and modest evidence indicates an effect of periodontal therapy in lowering these levels [10].

Technological developments have made it possible to detect and quantify a range of various biological markers in relatively small fluid specimens simultaneously. A high-throughput beadbased suspension array immunoassay system can assess up to 100 analytes in a sample volume of 25 to $50 \mu \mathrm{L}$ [11]. The quantification of multiple markers in one single sample can provide information on interactions that are inaccessible by studying single markers individually. The potential presence of very high values of several analytes at the same time was our particular focus, because such values tend to be disregarded as outliers in traditional single-marker analyses. A preliminary examination indeed suggested that among patients with untreated periodontitis, a few might exhibit high levels of several distinct inflammatory markers at the same time [12]. The presence and the disappearance of multiple extreme values may be biologically important in affected individuals, even if this does not concern all participants of a trial. The clustering of peaks has not been studied in periodontitis patients so far, and its clinical relevance is unknown. Studies on changes of serological inflammatory markers after periodontal therapy conducted so far rather looked at mean or median changes of single serological markers. The purpose of the present investigation was to further investigate this phenomenon by measuring a range of 24 analytes in the serum of periodontally diseased patients before and after nonsurgical periodontal therapy. This analysis is part of a large randomized clinical trial on the adjunctive effects of antibiotics given at different time points during periodontal therapy. For the present analysis, we focus on the outcomes at the 3-month reevaluation after initial therapy, and the differential effect of the antibiotics is not the primary subject here.

\section{Material and methods}

Participants and study design

Participants were recruited among patients seeking periodontal treatment or consulting for a routine dental checkup at the
School of Dental Medicine of the University of Geneva between April 2009 and August 2011. Forty patients with untreated moderate-to-advanced periodontitis [presence of at least four teeth with a probing pocket depth (PD) of $>4 \mathrm{~mm}$, clinical attachment loss of at least $2 \mathrm{~mm}$, and radiographic evidence of bone loss] participated in a single-center, randomized, placebo-controlled, parallel-group, and double-masked trial. In addition, 40 persons without evidence for past or present periodontal disease (absence of periodontal pockets with PD of $>3 \mathrm{~mm}$, absence of clinical attachment loss of $>1 \mathrm{~mm}$, and no radiographic evidence of bone loss) were included to provide a single venous blood sample. All participants were systemically healthy, within an age range of 25 70 years, and had at least 12 scorable teeth (not including third molars, teeth with orthodontic appliances, bridges, crowns, or implants). Exclusion criteria were systemic illnesses (i.e., diabetes mellitus, cancer, HIV, bone metabolic diseases, or disorders that compromise wound healing, radiation, or immunosuppressive therapy), pregnancy or lactation, systemic antibiotics taken within the previous 2 months, use of nonsteroid anti-inflammatory drugs, confirmed or suspected intolerance to 5-nitroimidazole derivatives or amoxicillin, and subgingival SRP or surgical periodontal therapy in the last year.

The Ethical Committee of the University Hospitals of Geneva, Geneva, Switzerland, approved the protocol. Research was conducted according to the principles outlined in the Declaration of Helsinki on human medical experimentation. Written informed consent was obtained from all participants.

\section{Clinical protocol}

Venous blood samples were drawn from all 80 participants. The 40 individuals with periodontitis were further examined and treated according to the clinical protocol outlined below. Blood samples were again obtained from the latter 12 weeks after therapy.

The clinical part of the study involved the examiner (AA), who obtained informed consent, organized the treatment sessions, and recorded all data, and the operator (NC), who provided the therapy. The therapist was unaware of the recorded data, except for the periodontal pocket chart that he needed to deliver the treatment.

The operator removed supragingival deposits and gave oral hygiene instructions and if necessary gave reinforcement of oral hygiene during recall visits. Once the subjects had reached an appropriate level of plaque control, they were scheduled to receive subgingival treatment within 1 month by the operator. Immediately before treatment, the examiner recorded the periodontal parameters. The operator treated the periodontally diseased teeth with thorough SRP to the depth of the pocket under local anesthesia. He first used ultrasonic instruments, then Gracey curettes, and finally irrigated the pockets with a $0.1 \%$ aqueous solution of chlorhexidine. 
Subjects were instructed to rinse the mouth twice daily during the next 10 days with $0.2 \%$ chlorhexidine. SRP was completed within $48 \mathrm{~h}$ and usually required two sessions.

At the end of the last session, each subject received a neutral package, prepared by the pharmacy of the Geneva University Hospital, containing either a test or placebo medication, and was advised to start with the drug regimen in the evening of the same day. Patients in the test group received $500 \mathrm{mg}$ metronidazole and $375 \mathrm{mg}$ amoxicillin, to be taken three times per day during 7 days, and patients in the control group received similar-looking placebos. The treatment was allocated using a computer-generated randomization list concealed to the patient, the clinical examiner, and the therapist. The examiner called the subjects after 1 week, and after 1 month. Medical history, any concomitant medication, and any adverse events or serious adverse events were recorded. The first posttreatment visit also served as a compliance control, as subjects were asked to return any medication that remained.

Immediately before (baseline (BL)) and 3 months after therapy (M3), the following clinical parameters were recorded on six sites of each tooth with a pocket of $>4 \mathrm{~mm}$ at baseline: Gingival Index (GI) [13], PD and recession (REC; positive if gingival margin located apical, negative if located coronal to the cementoenamel junction), bleeding upon probing (BOP), or suppuration.

\section{Blood sampling and processing}

Five milliliters of blood was obtained by venipuncture using tubes without anticoagulant. The specimens were immediately centrifuged at $1,300 \mathrm{~g}$ for $10 \mathrm{~min}$. Two milliliters of serum was stored at $-70{ }^{\circ} \mathrm{C}$ until the day of analysis.

We used a multiplex fluorescent bead-based immunoassay and the Bio-Plex 200 Suspension Array System (Bio-Rad Laboratories, Hercules, CA, USA) to assess two panels of serum markers. The first panel included the following 15 cytokines: IL-1 $\beta$, IL-1ra, IL-4, IL-6, IL-8, IL-10, IL-12, IL17 , basic FGF, G-CSF, GM-CSF, IFN- $\gamma$, MIP- $1 \beta$ VEGF, and TNF- $\alpha$ (kit M5000HIXIL, Bio-Rad Laboratories, Hercules, CA, USA). The second panel included the following nine acute-phase proteins: $\alpha 2 \mathrm{M}$, haptoglobin, CRP, serum amyloid $\mathrm{P}$ and $\mathrm{A}$, ferritin, fibrinogen, tissue plasminogen activator, and procalcitonin (kits 171A4009M and 171A4007M, Bio-Rad Laboratories, Hercules, CA, USA). The assays were performed following the manufacturer's instructions. Briefly, 96-well filter plates were pre-wetted with assay buffer, and the solution was aspirated from the wells using a vacuum filter. Microsphere beads coated with monoclonal antibodies against the 24 target analytes were added to the wells. Serum samples, controls, and standards were added in separate wells and incubated $30 \mathrm{~min}$ for the 15-plex panel and $1 \mathrm{~h}$ for the acute-phase protein panel. The wells were washed using the vacuum filter, and a mixture of biotinylated secondary antibodies was added. After incubation for $30 \mathrm{~min}$ and washing of the plates, streptavidin conjugated to the fluorescent protein phycoerythrin (streptavidin-PE) was added to the wells and incubated for $10 \mathrm{~min}$. After washing to remove the unbound reagents, the assay buffer was added to the wells, and the beads (minimum of 100 per analyte) were analyzed in the Bio-Plex 200 Suspension Array System. For the 15-plex panel, the detection limit of the assay was $1 \mathrm{pg} / \mathrm{ml}$. For the acute-phase protein panel, the limit of detection was $1 \mathrm{ng} / \mathrm{ml}$, with the exception of ferritin, procalcitonin, and tissue plasminogen activator, where the detection limit was $1 \mathrm{pg} / \mathrm{ml}$.

\section{Statistical analysis}

Because not all data were normally distributed, differences between placebo and test groups were analyzed using Wilcoxon matched-pair signed-rank test, a nonparametric test. Bio-Plex Manager 3.0 (Bio-Rad, Hercules, CA, USA) was used to analyze the readout. All analytes had a very skewed distribution, with most patients having normal values and a few patients having extreme values. Thus, we dichotomized each analyte into a normal versus extreme value. The cutoffs used to determine these peak values were defined as greater than the mean +2 standard deviations (SD) found in 40 unrelated periodontally healthy persons. If an analyte had a detection frequency below $25 \%$ in the healthy subjects, the cutoff was set at the respective detection level. To calculate mean values, a constant (0.1) was added to remove zero values. Proportions of peaks between treatment groups were compared using Fisher's exact tests. $p$ values of $<0.05$ were accepted for statistical significance. The statistical software R (version 2.15.1, The R Foundation for Statistical Computing, Vienna, Austria) was used for all analyses.

\section{Results}

The mean age of the healthy subjects was $37.2 \pm 11.0$, and of 26 females and 14 males, $10 \%$ were smokers. The mean age of the patients was $46.8 \pm 8.5$, and of 20 females and 20 males, $35 \%$ were smokers. In these patients, no significant differences were found between groups for the demographic variables.

The clinical results of the study before and 3 months after treatment are summarized in Table 1. All 40 enrolled patients with periodontitis could be followed up to M3. A total of 3,612 sites ( 6 on a total of 602 periodontally diseased teeth with a pocket of $>4 \mathrm{~mm}$ at BL; pockets of $>4 \mathrm{~mm}$ had a mean PD of $6.5 \mathrm{~mm}$ ) were clinically monitored at BL and M3. At $\mathrm{BL}$, differences between groups were not significant. In contrast, at M3, a significantly better primary clinical outcome was obtained in patients treated with full-mouth SRP plus amoxicillin and metronidazole compared to those receiving placebo. Those treated with antibiotics had fewer persisting 
Table 1 Clinical findings at baseline and after 3 months by treatment group. Data are means (standard deviation), $n=40$ participants

\begin{tabular}{|c|c|c|c|c|}
\hline \multirow[t]{2}{*}{ Parameters } & \multicolumn{2}{|l|}{ Baseline } & \multicolumn{2}{|l|}{ Month 3} \\
\hline & Placebo & Test & Placebo & Test \\
\hline Participants, $n$ & 21 & 19 & 21 & 19 \\
\hline $\mathrm{PD}, \mathrm{mm}$ & $4.4(2.0)$ & $4.7(2.3)$ & $2.9(1.3)$ & $2.6(1.0)$ \\
\hline $\mathrm{REC}, \mathrm{mm}$ & $0.9(1.0)$ & $0.6(0.8)$ & $1.2(1.3)$ & $1.0(1.0)$ \\
\hline BOP, $+\%$ & $90.7(29.0)$ & $89.9(30.1)$ & $37.8(49.5)^{*}$ & $27.5(44.7)^{*}$ \\
\hline GI, score & $0.9(0.6)$ & $1.0(0.6)$ & $0.2(0.4)$ & $0.2(0.4)$ \\
\hline $\begin{array}{c}\mathrm{PD}>4 \text { and } \mathrm{BOP}+ \\
n \text { sites/person }\end{array}$ & $32.2(19.8)$ & $37.2(34.8)$ & $6.8(7.8)^{*}$ & $3.3(5.1)^{*}$ \\
\hline
\end{tabular}

$P D$ probing pocket depth, $R E C$ recession, $B O P$ bleeding upon probing, $G I$ Gingival Index

${ }^{*} p<0.05$, difference between placebo and test

pockets of $>4 \mathrm{~mm}$ with BOP than those treated with placebo. In the patients of the placebo group, on average $6.8 \pm 7.8$ pockets of $>4 \mathrm{~mm}$ with BOP could still be found, whereas mere $3.3 \pm 5.1$ sites in this category were still detected in the test group.

A total of 120 serum samples were collected and analyzed for the presence of 15 cytokines and 9 acute-phase proteins. Table 2 shows the number of positive samples of all analytes in the 40 periodontally healthy subjects and, if detected in at least $25 \%$ of the specimens, the mean values, standard deviations, and mean $+2 \mathrm{SD}$. For the analytes detected in at least $25 \%$, the mean $+2 \mathrm{SD}$ served as a threshold to define peak values in periodontitis patients. Six cytokines could not be detected in any serum sample (IL-4, IL-17, b-FGF, G-CSF, GM-CSF, and IFN- $\gamma$ ), and three (IL-1 $\beta$, IL-6, and IL-10) were detected with a frequency below $25 \%$. For those nine cytokines, each positive reading in a patient was considered as a peak (i.e., the cutoff for inclusion was set at the respective level of detection).

Table 3 shows the incidence and range of peak values of 15 cytokines and 9 acute-phase proteins in 40 periodontitis patients before and after therapy. For example, four patients presented peak values for IL-1 $\beta$ at baseline and eight for IL1ra. These peak values ranged between 4.8 and 226.5 and between 21.7 and 127.3, respectively. At M3, none of the patients still presented a peak for IL- $1 \beta$, and only a single case still demonstrated a peak for IL-1ra.

For five biomarkers with high incidence of peaks of baseline, i.e., serum amyloid A, IL-1ra, IL-12, MIP-1 $\beta$, and procalcitonin, treatment significantly reduced the number of subjects with peaks $(p<0.05)$. Figure 1 shows a scatter plot for serum amyloid A, comparing the values before and after therapy for each subject. For one analyte, the number of subjects with peaks increased significantly after treatment (haptoglobin, $p=0.01$ ).
Table 2 Detection frequency of 15 cytokines and 9 acute-phase proteins in 40 periodontally healthy persons and, if detected in more than ten $(25 \%)$ cases, standard deviation (SD) and mean +2 SD. Mean +2 SD values served as a threshold to define peak values in periodontitis patients

\begin{tabular}{llll}
\hline Analyte & $N(\%)$ & Mean (SD) & Mean+2 SD (cutoff) \\
\hline IL-1 $\beta, p g / m l$ & $1(2.5)$ & - & $1^{\mathrm{a}}$ \\
IL-1ra, pg/ml & $35(87.5)$ & $4.3(6.9)$ & 18.1 \\
IL-4, pg/ml & $0(0.0)$ & - & $1^{\mathrm{a}}$ \\
IL-6, pg/ml & $2(5.0)$ & - & $1^{\mathrm{a}}$ \\
IL-8, pg/ml & 29 & $8.0(9.7)$ & 27.3 \\
IL-10, pg/ml & $9(22.5)$ & - & $1^{\mathrm{a}}$ \\
IL-12-p70, pg/ml & $40(100.0)$ & $29.5(16.9)$ & 63.3 \\
IL-17, pg/ml & $0(0.0)$ & - & $1^{\mathrm{a}}$ \\
basic-FGF, pg/ml & $0(0.0)$ & - & $1^{\mathrm{a}}$ \\
G-CSF, pg/ml & $0(0.0)$ & - & $1^{\mathrm{a}}$ \\
GM-CSF, pg/ml & $0(0.0)$ & - & $1^{\mathrm{a}}$ \\
IFN- $\gamma, \mathrm{pg} / \mathrm{ml}$ & $0(0.0)$ & - & $1^{\mathrm{a}}$ \\
MIP-1 $\beta, \mathrm{pg} / \mathrm{ml}$ & $40(100.0)$ & $143.0(58.0)$ & 259.1 \\
TNF- $\alpha, \mathrm{pg} / \mathrm{ml}$ & $18(45.0)$ & $14.0(30.2)$ & 74.3 \\
VEGF, pg/ml & $40(100.0)$ & $193.4(137.6)$ & 468.7 \\
$\alpha 2 \mathrm{M}, \mathrm{mg} / \mathrm{ml}$ & $40(100.0)$ & $1.9(0.7)$ & 3.3 \\
CRP, $\mu \mathrm{m} / \mathrm{ml}$ & $40(100.0)$ & $1.9(3.9)$ & 9.8 \\
Haptoglobin, mg/ml & $40(100.0)$ & $1.4(1.5)$ & 4.5 \\
Serum amyloid P, & $40(100.0)$ & $44.7(13.9)$ & 72.6 \\
$\mu \mathrm{g} / \mathrm{ml}$ & & & \\
Ferritin, ng/ml & $40(100.0)$ & $58.8(47.6)$ & 154.1 \\
Fibrinogen, $\mu \mathrm{g} / \mathrm{ml}$ & $40(100.0)$ & $2.5(0.6)$ & 3.8 \\
Procalcitonin, ng/ml & $36(90.0)$ & $2.2(1.4)$ & 5.0 \\
Serum amyloid A, & $40(100.0)$ & $3.4(2.8)$ & 8.9 \\
$\mu \mathrm{g} / \mathrm{ml}$ & & & \\
TP activator, ng/ml & $17(42.5)$ & $6.3(8.6)$ & 23.5 \\
\hline & & & \\
\hline
\end{tabular}

$I L-1 \beta$ interleukin-1 beta, IL-1ra interleukin-1 receptor antagonist, $I L-4$ interleukin-4, $I L-6$ interleukin-6, $I L-8$ interleukin-8, $I L-10$ interleukin-10, $I L-12$ interleukin-12, IL-17 interleukin-17, $b-F G F$ basic fibroblast growth factor, G-CSF granulocyte colony-stimulating factor, GM-CSF granulocyte macrophage colony-stimulating factor, $I F N-\gamma$ interferon gamma, $M I P-1 \beta$ macrophage inflammatory protein beta, $T N F-\alpha$ tumor necrosis factor alpha, $V E G F$ vascular endothelial growth factor, $\alpha 2 M$ alpha-2 macroglobulin, CRP C-reactive protein, TP tissue plasminogen

${ }^{\mathrm{a}}$ If an analyte was detected in less than $25 \%$, the detection level was used as a cutoff

We then calculated for each individual how many of the 24 analytes showed a peak value at baseline and how many peaks persisted after therapy, with or without antibiotics. As Fig. 2a shows, none of the 24 analytes were above threshold before treatment in ten participants. A single analyte showed a peak value in 11 patients, and two peaks were found in seven patients. In the remaining 12 patients, between three and ten analytes showed peak values. For individuals with two or more peaks, the mean values of PD and BOP were within the range of the other patients (PD, 4.41; BOP, 86.6). 
Table 3 Incidence (number and frequency) and range of peak values (readings above mean +2 SD of healthy persons) of $15 \mathrm{cy}-$ tokines and 9 acute-phase proteins in 40 periodontitis patients before and after therapy
IL-1 $\beta$ interleukin-1 beta, IL-1ra interleukin-1 receptor antagonist, $I L-4$ interleukin- $4, I L-6$ interleukin-6, IL-8 interleukin-8, IL-10 interleukin-10, IL-12 interleukin12, IL-17 interleukin-17, $b-F G F$ basic fibroblast growth factor, $G$-CSF granulocyte colony-stimulating factor, GM-CSF granulocyte macrophage colony-stimulating factor, $I F N-\gamma$ interferon gamma, $M I P-1 \beta$ macrophage inflammatory protein beta, $T N F-\alpha$ tumor necrosis factor alpha, $V E G F$ vascular endothelial growth factor, $\alpha 2 M$ alpha-2 macroglobulin, $C R P$ C-reactive protein, $T P$ tissue plasminogen

\begin{tabular}{|c|c|c|c|c|}
\hline \multirow[t]{2}{*}{ Analyte } & \multicolumn{2}{|l|}{ Baseline } & \multicolumn{2}{|l|}{ Month 3} \\
\hline & $N(\%)$ & Range & $N(\%)$ & Range \\
\hline IL-1 $\beta, p g / m l$ & $4(10.0)$ & $4.8-226.5$ & $0(0.0)$ & - \\
\hline IL-1ra, pg/ml & $8(20.0)$ & $21.7-127.3$ & $1(2.5)$ & 32.0 \\
\hline $\mathrm{IL}-4, \mathrm{pg} / \mathrm{ml}$ & $1(2.5)$ & 2.0 & $0(0.0)$ & - \\
\hline IL-6, pg/ml & $1(2.5)$ & 216.3 & $0(0.0)$ & - \\
\hline IL-8, pg/ml & $1(2.5)$ & 38.3 & $1(2.5)$ & 39.6 \\
\hline IL-10, pg/ml & $4(10.0)$ & $1.4-61.6$ & $5(12.5)$ & $1.4-4.9$ \\
\hline IL-12-p70, pg/ml & $5(12.5)$ & $66.7-125.8$ & $0(0.0)$ & - \\
\hline IL-17, pg/ml & $1(2.5)$ & 2.3 & $0(0.0)$ & - \\
\hline basic-FGF, pg/ml & $2(5.0)$ & $18.7-36.3$ & $1(2.5)$ & 11.9 \\
\hline G-CSF, pg/ml & $1(2.5)$ & 29.8 & $0(0.0)$ & - \\
\hline GM-CSF, pg/ml & $2(5.0)$ & $17.2-162.6$ & $0(0.0)$ & - \\
\hline $\mathrm{IFN}-\gamma, \mathrm{pg} / \mathrm{ml}$ & $3(7.5)$ & $13.2-5,087.0$ & $4(10.0)$ & $4.2-49.3$ \\
\hline MIP-1 $\beta, \mathrm{pg} / \mathrm{ml}$ & $6(15.0)$ & $260.5-791.2$ & $0(0.0)$ & - \\
\hline $\mathrm{TNF}-\alpha, \mathrm{pg} / \mathrm{ml}$ & $1(2.5)$ & 140.4 & $0(0.0)$ & - \\
\hline VEGF, pg/ml & $5(12.5)$ & $538.6-2,456.2$ & $3(7.5)$ & $525.7-5,518.7$ \\
\hline$\alpha 2 \mathrm{M}, \mathrm{mg} / \mathrm{ml}$ & $0(0.0)$ & - & $0(0.0)$ & - \\
\hline $\mathrm{CRP}, \mu \mathrm{g} / \mathrm{ml}$ & $4(10.0)$ & $13.4-52.6$ & $3(7.5)$ & $14.1-33.4$ \\
\hline Haptoglobin, mg/ml & $5(12.5)$ & $5.1-7.9$ & $16(40.0)$ & $5.8-9.4$ \\
\hline Serum amyloid $\mathrm{P}, \mu \mathrm{g} / \mathrm{ml}$ & $6(15.0)$ & $73.3-91.7$ & $10(25.0)$ & $74.6-138.1$ \\
\hline Ferritin, ng/ml & $8(20.0)$ & $212.2-505.8$ & $9(22.5)$ & $187.6-418.5$ \\
\hline Fibrinogen, $\mu \mathrm{g} / \mathrm{ml}$ & $1(2.5)$ & 3.9 & $0(0.0)$ & - \\
\hline Procalcitonin, ng/ml & $6(15.0)$ & $5.4-6.3$ & $0(0.0)$ & - \\
\hline Serum amyloid $\mathrm{A}, \mu \mathrm{g} / \mathrm{ml}$ & $14(35.0)$ & $9.7-20.0$ & $2(5.0)$ & $13.1-30.8$ \\
\hline $\mathrm{TP}$ activator, $\mathrm{ng} / \mathrm{ml}$ & $4(10.0)$ & $23.9-28.0$ & $0(0.0)$ & - \\
\hline
\end{tabular}

Figure $2 \mathrm{~b}$ shows the number of analytes with peak values per subject after therapy. Therapy did not significantly reduce the number of subjects having at least one peak (BL, 30 subjects; M3, 27 subjects; $p=0.62$ ). The number of subjects with four or more peaks, however, was greatly reduced (BL, 11 subjects; M3, 1 subject; $p=0.003$ ). With regards to the reduction of peaks, no specific benefit of adjunctive antibiotics could be seen. A single patient still showed eight peaks after treatment. This particular subject had eight peaks at BL also, was treated with antibiotics, and responded well to therapy as far as clinical parameters are concerned (mean PD at $\mathrm{BL}, 6.26 \mathrm{~mm}$; BOP at BL, $100 \%$; PD after therapy, $3.07 \mathrm{~mm}$; BOP after therapy, $39.9 \%$ ).

Table 4 shows the mean serum biomarker levels in periodontitis patients per treatment group at baseline and M3 of those 13 analytes with a detection frequency of $>75 \%$. The levels were significantly lower at M3 than those at baseline for most of these markers. Differences between groups were not significant.

There were no serious adverse events. The number of patients reporting a stomach upset was similar in both groups (three in the placebo group and three in the test group). One patient treated with antibiotics and three in the placebo group reported gastrointestinal problems, notably diarrhea. All other events were sporadic.

\section{Discussion}

We measured a range of 24 analytes in the serum of periodontally diseased patients before and 3 months after nonsurgical periodontal therapy and assessed to what extent levels of these analytes can be modified by treatment. A high-throughput technique was used to assess the expression profile of 15 cytokines and 9 acute-phase proteins in serum. The Bio-Plex 200 Suspension Array System is a flow-based dual-laser system that detects and measures molecules bound to the surfaces of fluorescent microspheres, thus providing a highly accurate analysis of serum, culture media, and other biological samples. Although the ELISA technique remains the gold standard for measuring inflammatory mediators, this new technology offers a number of advantages, such as the 
Fig. 1 Scatter plot for serum amyloid A. Each dot represents one case of 40 periodontally diseased subjects before and after therapy $(\mu \mathrm{g} / \mathrm{ml})$

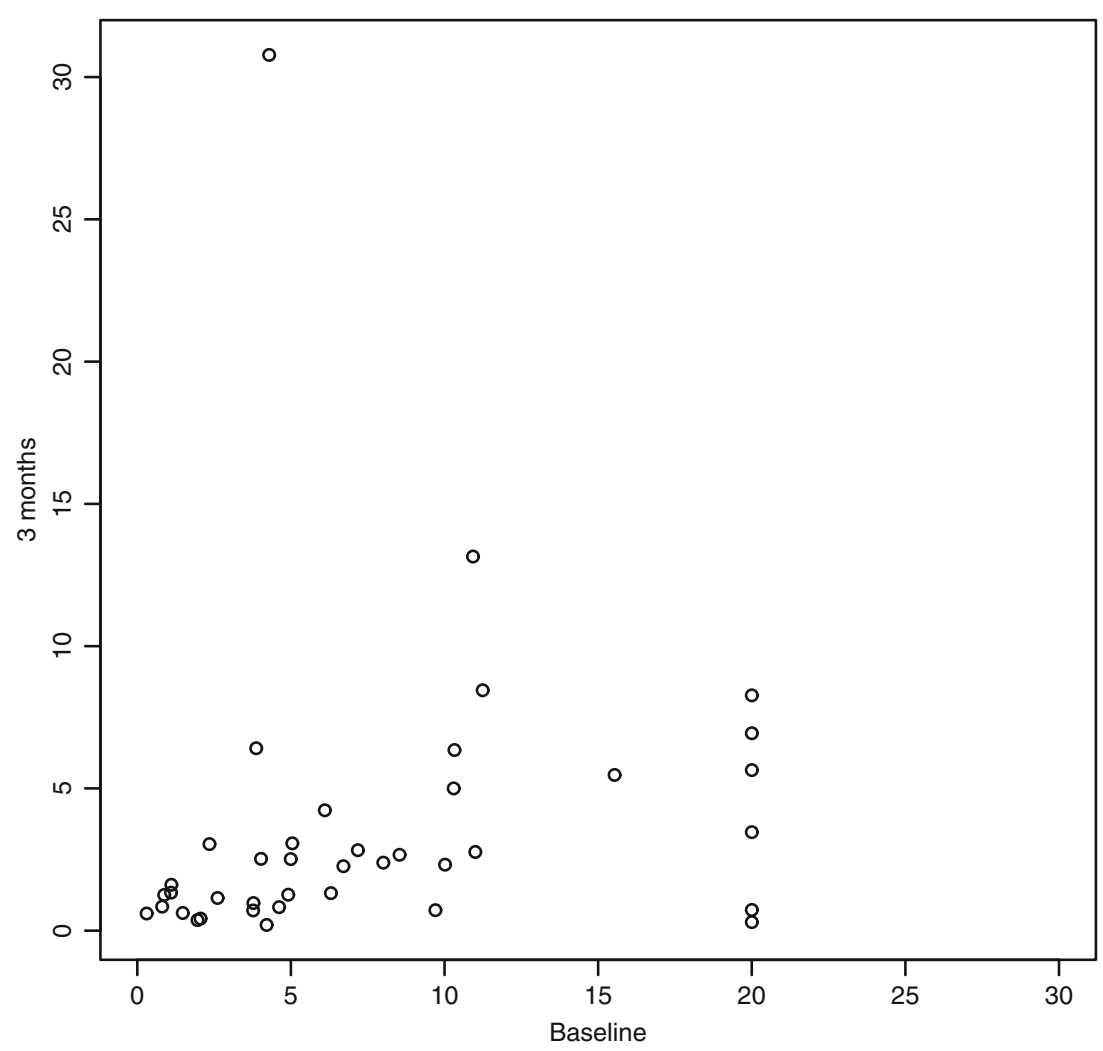

simultaneous determination of up to 100 different analytes in a single sample, less sample volume, ability to detect different proteins across a broad range of concentrations, and efficiency in terms of time and cost $[14,15]$. The cytokine multiplex assays were found to be comparable in sensitivity, accuracy, and reproducibility to ELISAs for the same analytes [16].

So far, most of the studies comparing levels of biochemical parameters between healthy subjects and periodontitis patients, or before and after therapy in diseased subjects, mean or median levels were presented, and comparisons were made using Mann-Whitney $U$ tests. Another way to analyze the overall systemic response to treatment is by calculating the summary inflammatory score (SIS); for each time point, a standardized within-person $z$ score is created for each analyte, and then by averaging the different analyte scores, the SIS is obtained for each patient at each time point. Using this approach, heterogeneous responses to periodontal therapy were found that correlated poorly with clinical and infectious markers of periodontitis [17].

Due to the distribution patterns of these markers found in preliminary analyses [7, 12], the present analysis focused on the incidence of high values of multiple analytes, rather than on mean or median changes or a summary score, assuming that multiple high values of several markers may be more consequential for systemic health than elevated mean levels of single markers. We show for the first time that some patients with untreated periodontitis indeed present high peaks for several inflammatory markers simultaneously and, furthermore, that simple, nonsurgical periodontal treatment with or without antibiotics has the potential to eliminate multiple peaks.

The mean +2 SD of each analyte, determined in 40 periodontally healthy persons unrelated to the trial cohort, served as a threshold to define peak values for the 40 periodontitis patients participating in the trial. Before therapy, 11 of the 40 patients enrolled in the trial exhibited levels above a threshold of four and up to ten inflammatory markers at the same time. After treatment, the number of subjects with four or more peaks was significantly reduced. Only a single patient remained with multiple peaks (eight analytes above the threshold). The fact that this person was the only one having also eight peaks at BL and responding well to therapy suggests that the reason for this unusual pattern was not periodontal disease. The number of subjects with peaks increased significantly after treatment for only one analyte (haptoglobin, $p=0.01$ ). This was probably due to random variability. We are aware of the limitations inferring a casual relationship between peak values of multiple inflammatory mediators in serum and the pathogenesis of periodontal disease, since this pattern was not recognized in every patient and we cannot determine its origin. However, several 
Fig. 2 Number of biomarker peaks per subject before (a) and after (b) therapy. Total number of biomarkers is 24 , and the number of cases is 40
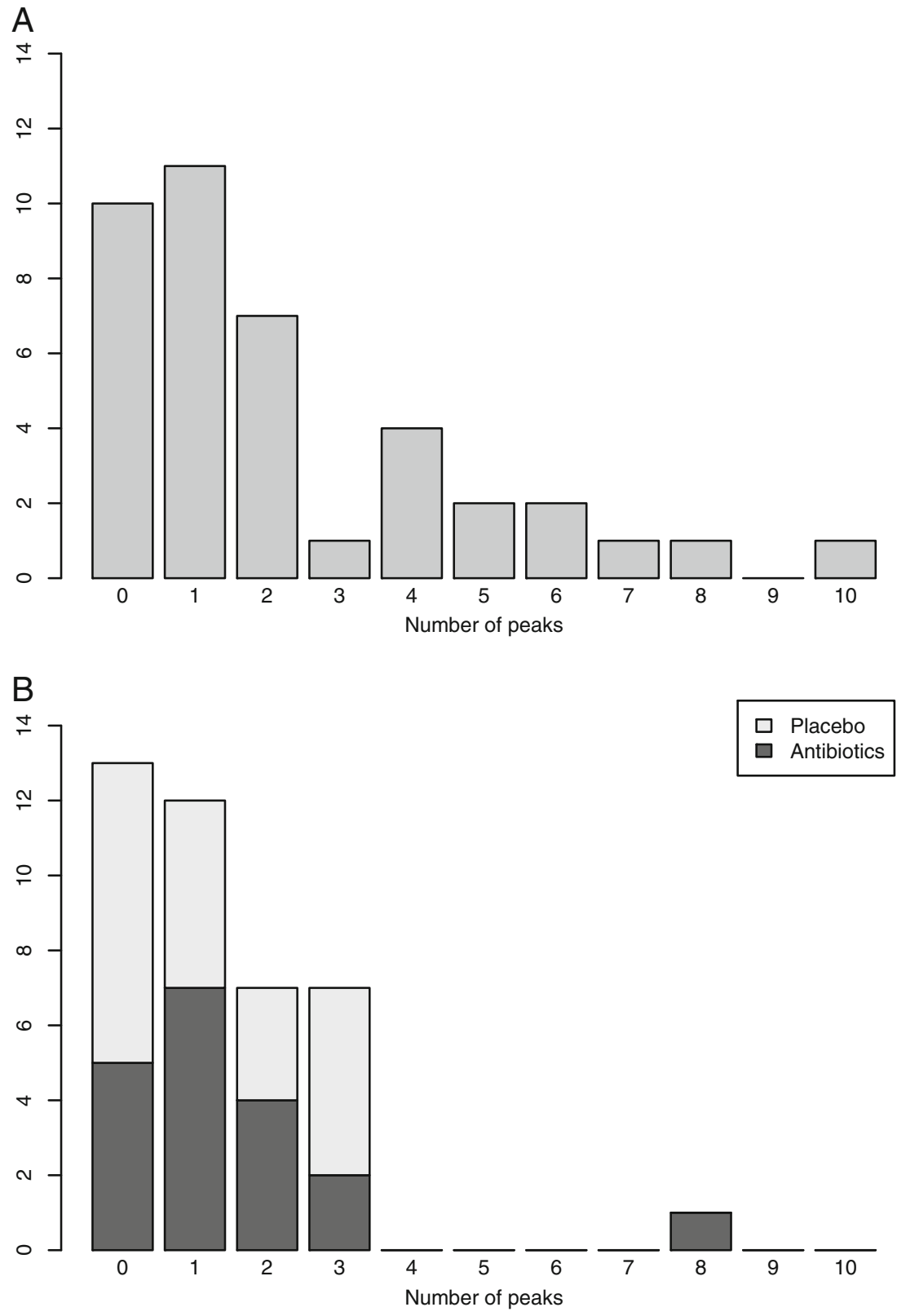

subjects belonging exclusively to the periodontitis group presented extreme values for an important number of analytes.

Conflicting results on the mean or median effects of periodontal therapy on systemic inflammation have been reported previously. Whereas a significant increase in plasma TNF- $\alpha$, CRP and IL-6 levels has been found immediately after nonsurgical periodontal therapy in some trials $[6,18,19]$, other studies found no changes in the serum levels of these mediators 3 months after therapy [20]. Six months after treatment, significant reductions in serum IL-6 and CRP were found especially in subjects who responded better than average to the delivered periodontal therapy [5]. However, in another study, the same group reported marked increases in the serum levels of CRP and serum amyloid A only $24 \mathrm{~h}$ after treatment
[21]. Responses of systemic inflammatory biomarkers were highly heterogeneous following periodontal therapy in a trial of another group where a large number of analytes was assessed simultaneously [17]. One third of the patients showed a marked reduction and one fourth a pronounced increase, and the remainder of the patients showed no change in systemic inflammation.

In the medical literature, IL-6 has been described as a reliable marker for systemic inflammation. Peak values of plasma IL-6 and other peripheral markers of inflammation were assessed in the first week of ischemic stroke in one study. Significant correlations between peak plasma IL-6 in the first week of ischemic stroke with both brain infarct volume and outcome at 3 months 
Table 4 Levels of 13 serum biomarkers with a detection frequency of $>75 \%$ in periodontitis patients per treatment group, at baseline and M3. Data are means (standard deviation), $n=40$ participants

\begin{tabular}{|c|c|c|c|c|c|}
\hline \multirow[t]{2}{*}{ Parameters } & \multicolumn{2}{|l|}{ Baseline } & \multicolumn{2}{|l|}{ Month 3} & \multirow{2}{*}{$\begin{array}{l}p \\
\text { BL-M3 }\end{array}$} \\
\hline & Placebo & Test & Placebo & Test & \\
\hline Participants, $n$ & 21 & 19 & 21 & 19 & \\
\hline IL-1ra, pg/ml & $12.6(29.3)$ & $5.8(8.5)$ & $0.5(1.2)$ & $2.0(7.3)$ & $<0.001$ \\
\hline IL-8, pg/ml & $5.7(8.6)$ & $6.7(6.3)$ & $5.6(9.5)$ & $6.1(7.3)$ & n.s. \\
\hline IL-12-p70, pg/ml & $25.8(20.9)$ & $30.0(30.6)$ & $5.8(4.6)$ & $8.4(6.1)$ & $<0.001$ \\
\hline $\mathrm{MIP}-1 \beta, \mathrm{pg} / \mathrm{ml}$ & $189.4(157.9)$ & $157.0(74.4)$ & $97.0(50.1)$ & $99.0(52.6)$ & $<0.001$ \\
\hline VEGF, pg/ml & $237.6(244.3)$ & $354.3(544.8)$ & $148.7(130.0)$ & $499.0(1,253.4)$ & 0.002 \\
\hline$\alpha 2 \mathrm{M}, \mathrm{mg} / \mathrm{ml}$ & $1.3(0.6)$ & $1.6(0.5)$ & $1.7(0.3)$ & $1.6(0.3)$ & 0.04 \\
\hline $\mathrm{CRP}, \mu \mathrm{g} / \mathrm{ml}$ & $5.7(8.1)$ & $6.4(11.7)$ & $4.6(4.4)$ & $5.1(7.6)$ & n.s. \\
\hline Haptoglobin, $\mathrm{mg} / \mathrm{ml}$ & $1.0(1.5)$ & $2.3(2.5)$ & $2.8(2.7)$ & $3.2(3.0)$ & 0.004 \\
\hline Serum amyloid $\mathrm{P}, \mu \mathrm{g} / \mathrm{ml}$ & $56.2(20.0)$ & $56.5(13.9)$ & $67.1(25.7)$ & $58.2(13.5)$ & 0.02 \\
\hline Ferritin, ng/ml & $117.0(166.9)$ & $109.4(122.6)$ & $99.0(128.4)$ & $97.0(107.0)$ & 0.002 \\
\hline Fibrinogen, $\mu \mathrm{g} / \mathrm{ml}$ & $2.6(0.9)$ & $2.3(1.0)$ & $1.9(0.6)$ & $1.8(0.6)$ & 0.001 \\
\hline Procalcitonin, $\mathrm{ng} / \mathrm{ml}$ & $2.5(2.1)$ & $2.3(1.8)$ & $0.5(0.6)$ & $0.5(0.8)$ & $<0.001$ \\
\hline Serum amyloid $\mathrm{A}, \mu \mathrm{g} / \mathrm{ml}$ & $8.9(7.0)$ & $6.5(5.2)$ & $3.6(3.5)$ & $3.7(6.7)$ & $<0.001$ \\
\hline
\end{tabular}

IL-1 ra interleukin-1 receptor antagonist, $I L-8$, interleukin-8, $I L-12$ interleukin-12, MIP-1 $\beta$ macrophage inflammatory protein beta, $V E G F$ vascular endothelial growth factor, $\alpha 2 M$ alpha-2 macroglobulin, CRP C-reactive protein, n.s. not significant

were demonstrated [22]. In a study including patients with or without periprosthetic infection following total hip and knee arthroplasty, normal values of IL-6 were defined as those below a cutoff of $10 \mathrm{pg} / \mathrm{ml}$ [23]. In our study, the threshold for IL-6 was similar $(14.3 \mathrm{pg} / \mathrm{ml})$. Only one patient had a value above this threshold before and none after therapy. However, in a large population of African children without or with malaria [24], the mean values were $54 \mathrm{pg} / \mathrm{ml}$ in healthy and $485 \mathrm{pg} / \mathrm{ml}$ in severely diseased subjects. Very high peak values were detected in some individuals of both groups. The same holds true for three other markers assessed in the same study (IL-1 $\beta$, IL-8, and IL-10), suggesting that the African cohorts may be exposed to additional immunological challenges even in the absence of malaria. On the other hand, our threshold values for IL-12 and TNF- $\alpha$ corresponded well to their mean values of healthy controls.

In our study, no correlation was found between the inflammatory mediator levels and the clinical parameters either before or after periodontal treatment. This is in agreement with other studies showing poor and inconsistent correlations between changes in the levels of inflammatory markers and changes in the clinical periodontal status after therapy, reduction of periodontal pathogens, and reduction of serum IgG antibody levels to periodontal microbiota $[7,17,25]$. It seems that serum levels of specific cytokines or acute-phase proteins do not reflect the clinical periodontal health status independently; preexisting susceptibility for systemic inflammation may influence the individual response. However, the reduction of most of the peak values, defined as greater than the mean $+2 \mathrm{SD}$ in periodontally healthy subjects, can be attributed to periodontal therapy. A marked increase of CRP has been reported $24 \mathrm{~h}$ after instrumentation [21], indicating that patients undergoing periodontal treatment experience short-term perturbations of systemic inflammation. Due to the timing of our trial, we could not corroborate the existence of short-term peaks of some of the acute-phase proteins, but even if they exist, it seems that they are short-lived, as they were undetectable at M3 in our study.

Systemic amoxicillin plus metronidazole significantly improved clinical outcomes of periodontal therapy, similar to previous studies [26]. No significant difference on the biologic response to therapy was, however, found between the two groups. We assume that the mechanical removal of pathogenic bacteria by SRP was sufficient to suppress peak values of inflammatory biomarkers.

\section{Conclusion}

Subjects with untreated periodontitis may show isolated high peaks for one or several inflammatory markers in serum. Nonsurgical periodontal treatment with or without antibiotics reduced most of these peak levels. 
Acknowledgments The Swiss National Science Foundation, grant no. 320030-122089, supported this study. We thank Céline Nicole-Ippolito for entering the data.

Conflict of interest The authors report no conflicts of interest related to this study.

\section{References}

1. Pussinen PJ, Paju S, Mantyla P, Sorsa T (2007) Serum microbial- and host-derived markers of periodontal diseases: a review. Curr Med Chem 14:2402-2412

2. Wick PA, Mombelli A, Pagano S et al (2013) Anti-apolipoprotein A-1 autoantibodies as biomarker for atherosclerosis burden in patients with periodontitis. J Periodontal Res 48:350-356

3. Linden GJ, Lyons A, Scannapieco FA (2013) Periodontal systemic associations: review of the evidence. J Periodontol 84:S8-S19

4. D'Aiuto F, Orlandi M, Gunsolley JC (2013) Evidence that periodontal treatment improves biomarkers and CVD outcomes. J Periodontol 84:S85-S105

5. D'Aiuto F, Parkar M, Andreou G et al (2004) Periodontitis and systemic inflammation: control of the local infection is associated with a reduction in serum inflammatory markers. J Dent Res 83:156-160

6. D'Aiuto F, Nibali L, Parkar M, Suvan J, Tonetti MS (2005) Shortterm effects of intensive periodontal therapy on serum inflammatory markers and cholesterol. J Dent Res 84:269-273

7. Teles FR, Teles RP, Martin L, Socransky SS, Haffajee AD (2012) Relationships among IL-6, TNF-alpha, adipokines, vitamin D and chronic periodontitis. J Periodontol 83:1183-1191

8. Rastogi P, Singhal R, Sethi A, Agarwal A, Singh VK, Sethi R (2012) Assessment of the effect of periodontal treatment in patients with coronary artery disease: a pilot survey. J Cardiovasc Dis Res 3:124-127

9. Lopez NJ, Quintero A, Casanova PA, Ibieta CI, Baelum V, Lopez R (2012) Effects of periodontal therapy on systemic markers of inflammation in patients with metabolic syndrome: a controlled clinical trial. J Periodontol 83:267-278

10. Paraskevas S, Huizinga JD, Loos BG (2008) A systematic review and meta-analyses on C-reactive protein in relation to periodontitis. J Clin Periodontol 35:277-290

11. Wong HL, Pfeiffer RM, Fears TR, Vermeulen R, Ji S, Rabkin CS (2008) Reproducibility and correlations of multiplex cytokine levels in asymptomatic persons. Cancer Epidemiol Biomarkers Prev 17: 3450-3456

12. Almaghlouth A, Giannopoulou C, Cancela J, Décaillet F, Cionca N, Mombelli A (2011) Multiplex analysis of serologic markers in periodontitis and health. J Dent Res 90(Special Issue B):2982
13. Löe H, Silness J (1963) Periodontal disease in pregnancy. I. Prevalence and severity. Acta Odontol Scand 21:533-551

14. Leng SX, McElhaney JE, Walston JD, Xie D, Fedarko NS, Kuchel GA (2008) ELISA and multiplex technologies for cytokine measurement in inflammation and aging research. J Gerontol A Biol Sci Med Sci 63:879-884

15. Elshal MF, McCoy JP (2006) Multiplex bead array assays: performance evaluation and comparison of sensitivity to ELISA. Methods $38: 317-323$

16. de Jager W, te Velthuis H, Prakken BJ, Kuis W, Rijkers GT (2003) Simultaneous detection of 15 human cytokines in a single sample of stimulated peripheral blood mononuclear cells. Clin Diagn Lab Immunol 10:133-139

17. Behle JH, Sedaghatfar MH, Demmer RT et al (2009) Heterogeneity of systemic inflammatory responses to periodontal therapy. J Clin Periodontol 36:287-294

18. Ide M, Jagdev D, Coward PY, Crook M, Barclay GR, Wilson RF (2004) The short-term effects of treatment of chronic periodontitis on circulating levels of endotoxin, C-reactive protein, tumor necrosis factor-alpha, and interleukin-6. J Periodontol 75:420-428

19. D'Aiuto F, Parkar M, Tonetti MS (2007) Acute effects of periodontal therapy on bio-markers of vascular health. J Clin Periodontol 34: 124-129

20. Yamazaki K, Honda T, Oda T et al (2005) Effect of periodontal treatment on the $\mathrm{C}$-reactive protein and proinflammatory cytokine levels in Japanese periodontitis patients. J Periodontal Res 40:53-58

21. Graziani F, Cei S, Tonetti M et al (2010) Systemic inflammation following non-surgical and surgical periodontal therapy. J Clin Periodontol 37:848-854

22. Smith CJ, Emsley HC, Gavin CM et al (2004) Peak plasma interleukin- 6 and other peripheral markers of inflammation in the first week of ischaemic stroke correlate with brain infarct volume, stroke severity and long-term outcome. BMC Neurol 4:2

23. Di Cesare PE, Chang E, Preston CF, Liu CJ (2005) Serum interleukin-6 as a marker of periprosthetic infection following total hip and knee arthroplasty. J Bone Joint Surg Am 87:1921-1927

24. Lyke KE, Burges R, Cissoko Y et al (2004) Serum levels of the proinflammatory cytokines interleukin-1 beta, IL-6, IL-8, IL-10, tumor necrosis factor alpha, and IL-12(p70) in Malian children with severe Plasmodium falciparum malaria and matched uncomplicated malaria or healthy controls. Infect Immun 72:5630-5637

25. Gorska R, Gregorek H, Kowalski J, Laskus-Perendyk A, Syczewska M, Madalinski K (2003) Relationship between clinical parameters and cytokine profiles in inflamed gingival tissue and serum samples from patients with chronic periodontitis. J Clin Periodontol 30:1046-1052

26. Cionca N, Giannopoulou C, Ugolotti G, Mombelli A (2009) Amoxicillin and metronidazole as an adjunct to full-mouth scaling and root planing of chronic periodontitis. J Periodontol 80:364-371 